\title{
Multilayer-based soft X-ray polarimeter at the Beijing Synchrotron Radiation Facility*
}

\author{
SUN Li-Juan(孙立娟) $)^{1,2}$ CUI Ming-Qi(崔明启 $)^{2,1} \quad$ ZHU Jie(朱杰) ${ }^{2} \quad$ ZHAO Yi-Dong(赵屹东 $)^{2}$ \\ ZHENG Lei(郑雷) ${ }^{2}$ WANG Zhan-Shan(王占山) ${ }^{3} \quad$ ZHU Jing-Tao(朱京涛) ${ }^{3}$ \\ ${ }^{1}$ State Key Laboratory of Nonlinear Mechanics, Institute of Mechanics, Chinese Academy of Sciences, Beijing 100190, China \\ ${ }^{2}$ Institute of High Energy Physics, Chinese Academy of Sciences, Beijing 100049, China \\ 3 Tongji University, Shanghai 200092, China
}

\begin{abstract}
A compact high precision eight-axis automatism and two-axis manual soft-ray polarimeter with a multilayer has been designed, constructed, and installed in 3W1B at the Beijing Synchrotron Radiation Facility (BSRF). Four operational modes in the same device, which are double-reflection, double-transmission, front-reflection-behindtransmission and front-transmission-behind-reflection, have been realized. It can be used for the polarization analysis of synchrotron radiation. It also can be used to characterize the polarization properties of the optical elements in the soft X-ray energy range. Some experiments with $\mathrm{Mo} / \mathrm{Si}$ and $\mathrm{Cr} / \mathrm{C}$ multilayers have been performed by using this polarimeter with good results obtained.
\end{abstract}

Key words: synchrotron radiation, multilayer, polarimeter, soft X-ray

PACS: 07.85 Qe, 07.60.Fs, $42.79 \mathrm{Ci} \quad$ DOI: $10.1088 / 1674-1137 / 37 / 7 / 078001$

\section{Introduction}

The polarization of soft X-ray synchrotron radiation is an exciting technique for biology, chemistry, physics, and materials science, such as soft X-ray circular dichroism spectroscopy [1] and magnetic linear dichroism spectroscopy [2] in ferro-, ferri- and paramagnetic compounds, measurement of the Faraday effect [3] and the Kerr effect [4] in soft X-rays, spin-polarized photoelectron [5] spectroscopy, etc. For these studies, it is very crucial to measure the actual polarization state of the incident light and characterize the polarized properties of optical elements. To make a complete polarization analysis of incident light, both a polarizer and an analyzer are needed. Intensity is detected while the two polarized elements rotate independently around the optical axis [6]. However, owing to the high absorption of materials for light, the polarized elements which are used in other energy ranges fail to polarize in the soft $\mathrm{X}$-ray region. So, multilayers are chosen as polarized elements in the light of their interference structures. At present, there are several types of soft X-ray polarimeter, e.g., the polarizer and the analyzer are reflectors (i.e. double reflections) [7], the two polarized elements are transmitters (i.e. double-transmissions) [8], and the polarizer is a transmitter and the analyzer is a reflector (i.e. front-transmission-behind-reflection) [9, 10], etc. Nowadays, for a reflection polarizer, the multilayer can have high reflectivity, however, the direction of the incident light needs to be changed during experiments. So it is difficult to align the optical axis and insert other elements. For transmission polarizer optics, the direction of the light does not need to be changed. It is easy to align the optical axis, considerably simplify the whole system, and insert optical elements. However, it is difficult to improve the fabrication of the transmission multilayer. To optimize the devices, four operational modes can be performed in this polarimeter. They are double-reflection, doubletransmission, front-transmission-behind-reflection, and front-reflection-behind-transmission (i.e. the polarizer is a reflector and the analyzer is a transmitter). In this paper, the compact high-precision eight-axis automatism and two-axis manual high vacuum compatible polarimeter in soft X-ray range is presented. Some good results using this polarimeter with various polarized elements are shown.

\section{The multilayer optical elements}

As a reflection polarizer, a multilayer functions by positioning the multilayer interference peak near the Brew-

Received 18 September 2012, Revised 10 December 2012

* Supported by NSFC(11075176)

1) E-mail: cuimq@ihep.ac.cn

(C) 2013 Chinese Physical Society and the Institute of High Energy Physics of the Chinese Academy of Sciences and the Institute of Modern Physics of the Chinese Academy of Sciences and IOP Publishing Ltd 
ster angle [11]. This results in strong reflection of the s-component relative to the p-component. Here the scomponent of reflective intensity can be large, while the p-component is very small. So the grazing incident angle of the polarizer is named the quasi-Brewster angle of the multilayer mirrors. In addition, a multilayer can also be used as a linear transmission polarizer. Near the quasi-Brewster angle of a multilayer mirror, the strong reflection of the s-component to the p-component, results in strong transmission of the p-component relative to the s-component [12]. In the design and fabrication of a reflective polarizer, both the s-reflectivity and the extinction ratio of s-reflective intensity to p-reflective intensity, $I_{\mathrm{s}} / I_{\mathrm{p}}$ where $I_{\mathrm{s}}$ and $I_{\mathrm{p}}$ are the reflective intensities for s- and p-polarized radiation respectively, should be maximized. To optimize a transmission polarizer, the p-transmissive intensities should be maximized, however, the suppression ratio of s-transmissive intensity to p-transmissive intensity, $I_{\mathrm{s}} / I_{\mathrm{p}}$ where $I_{\mathrm{s}}$ and $I_{\mathrm{p}}$ are the transmissive intensities for $\mathrm{p}$ - and s-polarized component respectively, should be minimized.

The reflective multilayers are deposited on silicon substrates by using an ultrahigh vacuum DC magnetron sputtering facility in Ar (purity 99.999\%) at room temperature. The transmission multilayers are deposited on $100 \mathrm{~nm} \mathrm{Si}_{3} \mathrm{~N}_{4}$ substrate with a window size of $5 \mathrm{~mm} \times$ $5 \mathrm{~mm}$. All multilayer are measured using a diffractometer with $\mathrm{Cu} \mathrm{K}_{\alpha}$ radiation.

\section{The soft X-ray polarimeter}

The polarimeter is installed at $4.2 \mathrm{~m}$ behind the exit slit of Beamline $3 \mathrm{~W} 1 \mathrm{~B}$ of BSRF. The beamline is a windowless fully Ultra-high vacuum (UHV) soft X-ray beamline [13]. It is a monochromatic focusing soft Xray beamline from a permanent magnetic wiggler source. The photon energy region is from $50 \mathrm{eV}$ to $1600 \mathrm{eV}$. Typically, the photon flux and the energy resolution $(\Delta E / E)$ are $1 \times 10^{10}$ photos $\mathrm{mA}^{-1} \cdot \mathrm{s}^{-1}$ and $8 \times 10^{-4}-4 \times 10^{-3}$ at 50 $1600 \mathrm{eV}$, respectively.

The chamber of the polarimeter links to the beamline by UHV ferrofluidics feedthrough on the upstream. It can be rotated around the axis of the incident beam. The chamber is supported on gimbals and installed in a rigid bench which can move in three dimensions. Fig. 1 shows a schematic diagram of the polarimeter. The system is consisted of a set of $x-y$ exact translation stages systems (C), $I_{0}$ detector, polarizer assembly (P) (includes rotation of the grazing incident angle $\theta_{\mathrm{p}}$ and the azimuth angle $\alpha$ ), moving rocker $(\mathrm{R})$, sample stage $(\mathrm{S})$, analyzer assembly (A) (includes rotation of the grazing incident angle $\theta_{\mathrm{A}}$ and the azimuth angle $\beta$ ), main detector (D), data collectors and control systems. Table 1 shows its parameters. The device is equipped with $\mathrm{HV}$-compatible stepper motors except for the sample stage and the az- imuth of the polarizer. The device includes a two doubleangle compound framework with reflection or transmission polarizing elements when the polarizer/analyzer rotates independently around the axis of the light (i.e. the azimuth angle $(\alpha$ and $\beta))$ : one is the grazing incident angle $\theta_{\mathrm{P}}$ of the polarizer and the moving rocker $2 \theta_{\mathrm{P}}\left(\theta_{\mathrm{R}}\right)$, the other is the grazing incident angle $\theta_{\mathrm{A}}$ of the analyzer and the detector $2 \theta_{\mathrm{A}}\left(\theta_{\mathrm{D}}\right)$. The azimuth angle $\alpha$ of the polarizer around the incident beam axis can be set manually by rotating a rocker outside the chamber (the rocker is not shown in Fig. 1), and the azimuth $\beta$ of the analyzer is rotated about the beam axis from the polarizer by rotating the stage of the analyzer. For the reflection polarizer, when the grazing incident angle of the polarizer is $\theta_{\mathrm{P}}, \theta_{\mathrm{R}}$ the angle of the moving rocker can be rotated into $2 \theta_{\mathrm{P}}$, meeting the relation $\theta_{\mathrm{P}}-2 \theta_{\mathrm{P}}$. However, for the transmission polarizer, when the grazing incident angle of the polarizer is $\theta_{\mathrm{P}}$, the angle of moving rocker is $0^{\circ}$. Similarly, for the reflection analyzer, when the grazing incident angle of the analyzer is set to $\theta_{A}$, the grazing incident angle $\theta_{\mathrm{D}}$ of the detector can be rotated into $2 \theta_{\mathrm{A}}$. For the transmission analyzer, when the grazing incident angle of the analyzer is $\theta_{\mathrm{A}}$, the grazing incident angle $\theta_{\mathrm{D}}$ of the detector is kept $0^{\circ}$. Furthermore, the detector can be moved in a detective plane and the moving range is $\pm 3 \mathrm{~mm}$.



Fig. 1. A schematic picture of the polarimeter: 1, chamber; 2, azimuthal angle $\alpha$ of polarizer; 3 , collimator translation $x-y$ stage; $4, I_{0}$ detector; 5 , polarizer; 6 , moving rocker; 7 , sample stage; 8 , rotational stage to change azimuth angle of analyzer; 9, analyzer; 10, main detector.

Since all the operations must be done under vacuum conditions in the soft X-ray region, the chamber volume is limited. In addition, the size of the detector must be as small as possible with high efficiency and uniformity in the sensitivity over the whole range of the detective area. 
Table 1. The parameter of the rotation angle for the polarimeter.

\begin{tabular}{cccc}
\hline & symbol & range of angle $/\left(^{\circ}\right)$ & resolution $/\left(^{\circ}\right)$ \\
\hline polarizer: azimuth angle & $\alpha$ & $-180-190$ & 0.1 \\
incident angle of the polarizer & $\theta_{\mathrm{P}}$ & $0-90$ & 0.015 \\
azimuth angle of the analyzer & $\beta$ & $-180-190$ & 0.01 \\
incident angle of the analyzer & $\theta_{\mathrm{A}}$ & $0-90$ & 0.02 \\
moving rocker incident angle & $\theta_{\mathrm{R}}$ & $0-140$ & 0.03 \\
main detector & $\theta_{\mathrm{D}}$ & $0-140$ & 0.04 \\
incident angle of the sample stage & $\theta_{\mathrm{S}}$ & $0-90$ & 0.1 \\
\hline
\end{tabular}

A micro-channel plate (MCP) meets these demands. In order to improve the gain and prevent ion feedback, a chevron arrangement of two MCPs with the diameter of detective area $\phi 18.8 \mathrm{~mm}$ is used. The applied potential of the MCP detector is fixed in the measurements so that the gain of MCP detector is consistent. In addition, a photodiode also is used as the main detector. The signal of main detector is measured by an electrometer (6517A, KEITHLEY, USA). A computer is used for the data acquisition and all of the controlling units.

\section{Experiment}

For the reflective optical elements, the quasi-Brewster angle of the multilayer mirrors $\theta_{\mathrm{PqB}}$ is that both the s-reflective intensity and the extinction ratio of s- and p-reflective intensity, $I_{\mathrm{s}} / I_{\mathrm{p}}$ take the maximum at $\theta_{\mathrm{PqB}}$. Firstly, $I_{\mathrm{s}}$, the reflective intensity s component of incident light as a function of the grazing incident angles of the polarized element is measured by the double-angle scanning $(I-\theta)$ method. When $I_{\mathrm{s}}$ is measured, at a fixed energy, the azimuth angle $\alpha$ of the polarizer is $0^{\circ}$, the grazing incident angle of the polarizer and the moving rocker (the angle of the detector at $0^{\circ}$ ) are set to $\theta_{\mathrm{P}}$ and $2 \theta_{\mathrm{P}}$, respectively. Secondly, $I_{\mathrm{p}}$ is measured when $\alpha$ is $90^{\circ}$. So, the extinction ratio of s- and p-reflective intensity $I_{\mathrm{s}} / I_{\mathrm{p}}$ is obtained. Then, $\theta_{\mathrm{PqB}}$ of the polarizer is confirmed.

In addition, for the transmissive polarizing elements, the quasi-Brewster angle of the multilayer mirrors is that both the p-reflective intensity and the extinction ratio of p- and s-transmission intensity, $I_{\mathrm{p}} / I_{\mathrm{s}}$, take the maximum at $\theta_{\mathrm{PqB}}$. When the azimuth angle $\alpha$ of the polarizer is $0^{\circ}$, the transmissive intensity $\left(I_{\mathrm{p}}\right)$ is relative to the $\mathrm{p}$ component of the incident light.

In measurement, a $\mathrm{Si}$ filter is used. The Si filter (thickness: $400 \mathrm{~nm}$ ) has a transmission of $30 \%-50 \%$ over the range $55-95 \mathrm{eV}$.

\section{$5 \quad$ Results and discussions}

\subsection{The double-reflection mode}

Firstly, the quasi-Brewster angle $\theta_{\mathrm{PqB}}$ of the polarizer $(\mathrm{Cr} / \mathrm{C}, N=100 ; d=4.318 \mathrm{~nm}$ ) is measured at $206 \mathrm{eV}$ by using the method described in Section 4.2. The angle $45.2^{\circ}$ is $\theta_{\mathrm{PqB}}$ of the polarizer. Secondly, the grazing incident angle of the polarizer is set at $45.2^{\circ}$, and the moving rocker is set to $90.4^{\circ}$. The azimuth angle $\alpha$ of the polarizer is $0^{\circ}$. Then, the quasi-Brewster angle $\theta_{\mathrm{PqB}}$ of the analyzer is measured. Fig. 2(a) shows the reflective intensities of A $(\mathrm{Cr} / \mathrm{C}, N=100 ; d=4.35 \mathrm{~nm})$ for sand p-components and extinction ratio $I_{\mathrm{s}} / I_{\mathrm{p}}$ with reflection multilayer at $206 \mathrm{eV}$. Both the s-reflective intensity and the extinction ratio $I_{\mathrm{s}} / I_{\mathrm{p}}$ take the maximum at the grazing incident angle $\theta_{\mathrm{A}}$ of the analyzer $45.2^{\circ}$.
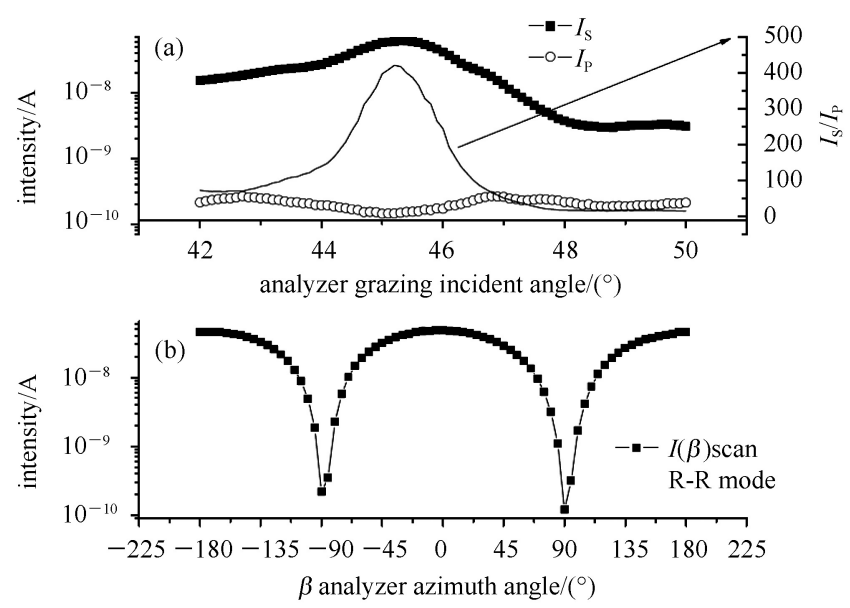

Fig. 2. Signals measured in double-reflections mode with $\mathrm{Cr} / \mathrm{C}(N=100$; polarizer, $d=4.318 \mathrm{~nm}$; analyzer, $d=4.35 \mathrm{~nm})$ reflection multilayer @ 206 eV. (a) double angle scanning; (b) azimuth angle scanning.

Figure 2(b) shows the curve of the azimuth angle of the analyzer scanning $(I-\beta)$ at the energy of $206 \mathrm{eV}$, corresponding to a rotation range from $-180^{\circ}$ to $190^{\circ}$. The grazing incident angles of the polarizer and the analyzer $\left(\theta_{\mathrm{P}}\right.$ and $\left.\theta_{\mathrm{A}}\right)$ are fixed at $45.2^{\circ}$, which are the quasiBrewster angle of the polarizing elements. During the azimuth rotation, $\theta_{\mathrm{P}}-\theta_{\mathrm{R}}$ and $\theta_{\mathrm{A}}-\theta_{\mathrm{D}}$ are satisfied with a two double-angle relation, respectively. A maximum signal is obtained for the azimuth of the analyzer $\beta=0$, $\pm 180^{\circ}$, which corresponds to s geometry; $\beta= \pm 90^{\circ}$ corresponds to $\mathrm{p}$ geometry. In order to show the difference of the minimum signal, Fig. 2(b) adopts the logarithm 
coordinate in $y$ direction. The results show that there is some difference at $\beta @ \pm 90^{\circ}$. However, the fit results are little affected by this difference. According to the data in Fig. 2(b), the degree of linear polarization of the beam reflected from the polarizer is more than 0.995 .

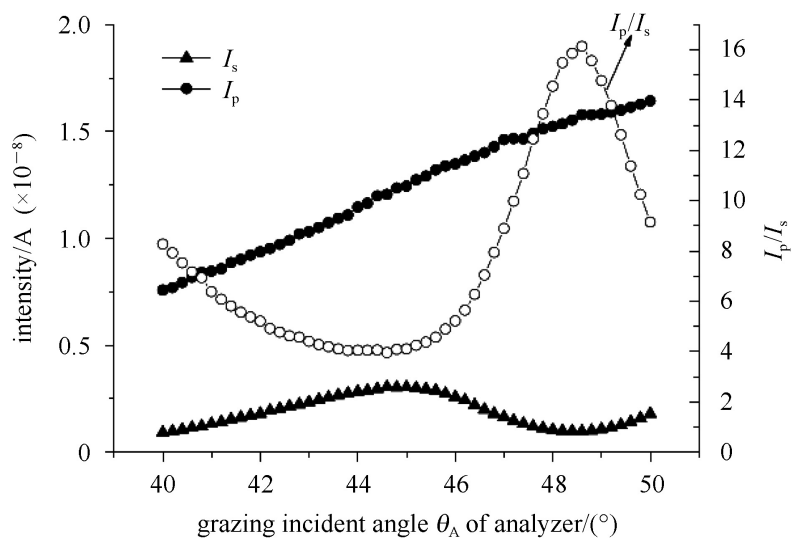

Fig. 3. Signals with Mo/Si @92.5 eV measures in front-reflection-behind-transmission mode.

\subsection{The front-reflection-behind-transmission mode}

Firstly, the quasi-Brewster angle $\theta_{\mathrm{PqB}}$ of the polarizer $(N=25 ; d=9.5 \mathrm{~nm}, \mathrm{Mo} / \mathrm{Si})$ is $47.2^{\circ}$ at $92.5 \mathrm{eV}$ by using the method in Section 4. Secondly, the grazing incident angles of the polarizer and the moving rocker are set to $47.2^{\circ}$ and $94.4^{\circ}$, respectively, while the azimuth $\alpha$ of the polarizer is set $0^{\circ}$. The grazing incident angle of the analyzer is set to $\theta_{\mathrm{A}}$, and the grazing incident angle $\theta_{\mathrm{D}}$ of the detector is kept to $0^{\circ}$. Then, the quasi-Brewster angle $\theta_{\mathrm{PqB}}$ of the analyzer is measured. Fig. 3 shows the transmissive intensities of the analyzer, $(N=25$; non-periodic wideband multilayer, $\mathrm{Mo} / \mathrm{Si}$ ) for $\mathrm{p}$ - and s-components and extinction ratio $I_{\mathrm{s}} / I_{\mathrm{p}}$ at $92.5 \mathrm{eV}$ in front-reflectionbehind-transmission modes. The $p$-component transmissive intensity, $I_{\mathrm{p}}$, and the ratio $I_{\mathrm{p}} / I_{\mathrm{s}}$ take the maximum value when the grazing incident angle $\theta_{\mathrm{A}}$ of the analyzer is $48.6^{\circ}$. So this angle is named the quasi-Brewster angle of the analyzer.

\subsection{The front-transmission-behind - reflection mode}

The quasi-Brewster angle $\theta_{\mathrm{PqB}}$ of a polarizer $(d=$ $9.715 \mathrm{~nm} ; N=21, \mathrm{Mo} / \mathrm{Si}$ ) is $47.2^{\circ}$ at $99 \mathrm{eV}$ by using the method in Section 4. The grazing incident angle of the polarizer is set to $47.2^{\circ}$, and the grazing incident angle of the moving rocker is set to $0^{\circ}$. For the reflective analyzer, the grazing incident angle of the analyzer is set to $\theta_{\mathrm{A}}$, and the grazing incident angle $\theta_{\mathrm{D}}$ of the detector can be rotated into $2 \theta_{\mathrm{A}}$. Fig. 4 shows the reflective intensities of the analyzer $(d=9.5 \mathrm{~nm} ; N=25, \mathrm{Mo} / \mathrm{Si})$ for $\mathrm{p}$ - and s-components and extinction ratio $I_{\mathrm{s}} / I_{\mathrm{p}}$ at $99 \mathrm{eV}$ when the azimuth angle $\alpha$ of the polarizer is $90^{\circ}$. Both the s-reflective intensity and the extinction ratio $I_{\mathrm{s}} / I_{\mathrm{p}}$ take the maximum with $\theta_{\mathrm{A}}$ at $47.2^{\circ}$. So the quasi-Brewster angle of the analyzer is $47.2^{\circ}$.

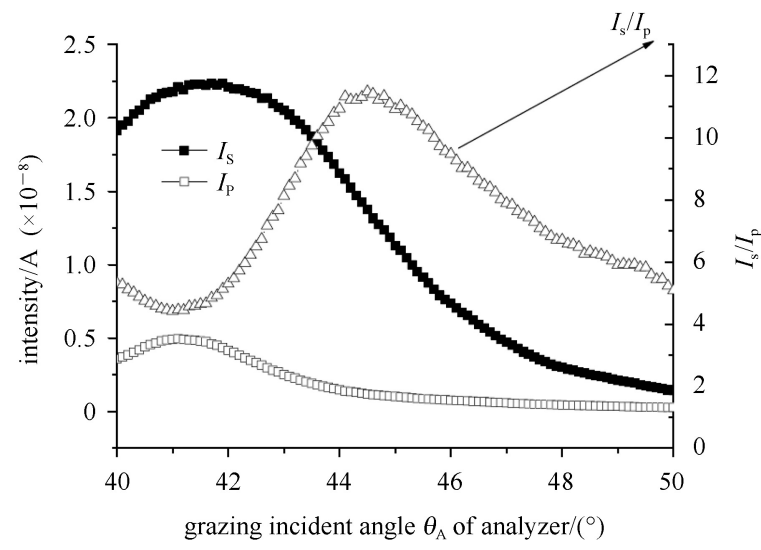

Fig. 4. Signals measured with $\mathrm{Mo} / \mathrm{Si}$ at $99 \mathrm{eV}$ in front-transmission-behind-reflection mode.

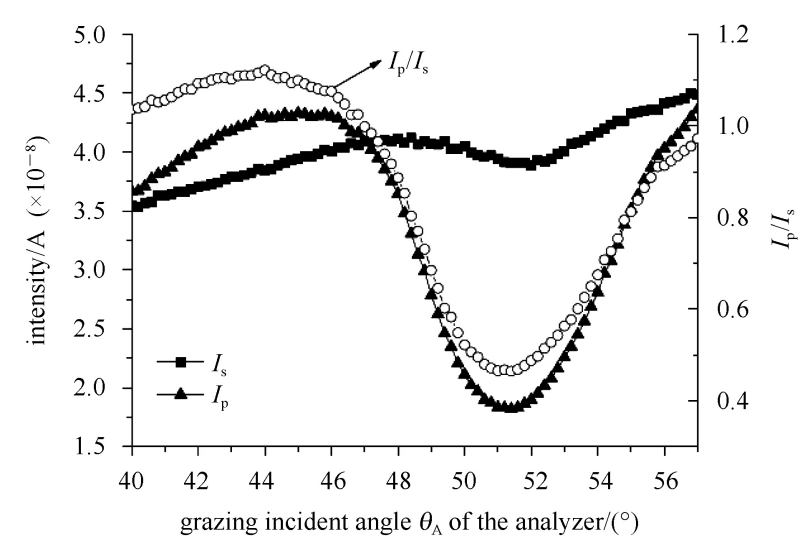

Fig. 5. Signals measured in double transmission mode with $\mathrm{Mo} / \mathrm{Si}$ at $92.5 \mathrm{eV}$.

\subsection{The transmission multilayer in double transmission mode}

The quasi-Brewster angle $\theta_{\mathrm{PqB}}$ of the polarizer ( $N=21$; non-periodic wideband multilayer, $\mathrm{Mo} / \mathrm{Si}$ ) is $51.5^{\circ}$ at $92.5 \mathrm{eV}$ by using the method in Section 4 . The grazing incident angle of the polarizer is set to $51.5^{\circ}$, and the grazing incident angle of the moving rocker is set to $0^{\circ}$. For the transmission analyzer, when the grazing incident angle of the analyzer is $\theta_{\mathrm{A}}$, the grazing incident angle $\theta_{\mathrm{D}}$ of the detector is kept $0^{\circ}$. Fig. 5 shows the transmissive intensities of the analyzer (nonperiodic wideband multilayer, $N=25, \mathrm{Mo} / \mathrm{Si}$ ) for $\mathrm{p}$ - and s-components with transmission multilayer at $92.5 \mathrm{eV}$ in double-transmission mode. Fig. 6 also shows that the extinction ratio $I_{\mathrm{s}} / I_{\mathrm{p}}$ takes the minimum value when a grazing incident angle $\theta_{\mathrm{A}}$ of the analyzer is $51.4^{\circ}$. So this angle is selected as the quasi-Brewster angle of the analyzer. 


\section{Conclusions}

Measurements of polarization are important for polarization-sensitive studies. This polarimeter with multilayers can perform four operational modes based on a variety of optical elements. The high precision eightaxis electromotion and two manual-axes HV-compatible polarimeter can be used as a device for the analysis of the polarization characteristics of the beamline at the Beijing Synchrotron Radiation Facility. It may be also used to characterize the reflection and the transmission properties or the polarization and the phase-retarding properties for various optical elements. Transmission or reflection polarizing elements enable appropriate control of the polarization of the probe beam. Furthermore, when either the polarizer or the analyzer is removed, this instrument can be used as a versatile reflectometer. With this instrument, the multilayer properties and full polarization measurement of synchrotron radiation [14] were characterized. And magneto-optical Faraday rotation around the $\mathrm{M}_{2,3}$ edges of $\mathrm{Ni}$ film $(d=31 \mathrm{~nm})$ was also measured $[15,16]$.

The authors would like to express their sincere thanks to the staff of the Soft X-ray Group of BSRF.

\section{References}

1 Eimüller T, Kato T, Mizuno T et al. Appl. Phys. Lett., 2004, 85(12): 2310

2 Scholl A, Stöhr J, Lüning J et al. Scinece, 2000, 287: 10141016

3 Mertins H-Ch, Schäfers F, Cann X Le et al. Phys. Rev. B, 2000, 61(2): R874-R877

4 Guedes I, Grimsditch M, Metlushko V et al. Phys. Rev. B., 2003, 67(2): 024428

5 Zaharko O, Mertins H-Ch, Schäfers F. Nucl. Instrum. Methods A, 2001, 467-468: 1419-1422

6 Kortright J B, Rice M, Kim S K et al. J. Magn. Magn. Mater., 1999, 191(1-2): 79-89

7 Kimura H, Yamamoto M, Yanagihara M et al. Rev. Sci. Instrum., 1992, 63(1): 1379-1380

8 Haga T, Utsumi Y, Itabashi S SPIE Conference on X-ray and
Ultraviolet Spectroscopy, 1998, 3443: 117-119

9 Schäfers F, Mertins H-Ch, Gaupp A S et al. Appl. Opt., 1999, 38(19): 4074-4088

10 WANG H, Dhesi S S, Maccherozzi F et al. Rev. Sci. Instrum., 2011, 82(12): 123301

11 Gluskin E S, Gaponov S V, Dhez P et al. Nucl. Instrum. Methods A, 1986, 246(1-3): 394-396

12 Kortright J B. Proc. SPIE, 1993, 2010: 160-167

13 CUI Ming-Qi, CUI Cong-Wu, ZHAO Yi-Dong et al. Atomic Energy Science and Technology, 1998, 32(6): 492-498 (in Chinese)

14 SUN Li-Juan, CUI Ming-Qi, ZHU Jie et al. HEP \& NP, 2007 31(4): 400-404 (in Chinese)

15 YAN Fen, CUI Ming-Qi, CHEN Kai et al. Acta. Phys. Sin. 2008, 57(5): 2865-2865 (in Chinese)

16 CHEN Kai, CUI Ming-Qi, YAN Fen et al. Chin. Phys. Lett. 2008, 25(3): 1110-1112 